\section{Prozessqualität in der stationären Suchtrehabilitation}

Zusammenfassung: Im Rahmen des Reha-Qualitätssicherungsprogramms der gesetzlichen Rentenversicherung wurde ein auf die spezifischen Belange der Suchterkrankung zugeschnittenes Verfahren zur Beurteilung der Prozessqualität entwickelt. Das Peer Review-Verfahren nutzt dabei als Informationszugang zu den Prozessabläufen die nach einem einheitlichen Raster erstellten Entlassungsberichte der Patienten. Pro Klinik zufällig ausgewählte Berichte werden von erfahrenen und geschulten Fachkollegen (Peers) auf der Grundlage einer detaillierten Liste qualitätsrelevanter Prozessmerkmale und eines Manuals bewertet. Die bereits bei anderen rehabilitativen Indikationen erprobte Methode des Peer-Reviews zeigt bei der empirischen Überprüfung auf der messtechnischen Ebene auch gute bis zufriedenstellende Kennwerte für den Bereich der Abhängigkeitserkrankungen. Das Verfahren erlaubt eine differenzierte Beschreibung und Analyse der Stärken und Schwächen bei der Durchführung der rehabilitativen Behandlungsmaßnahmen. Ein ausgearbeitetes Rückmeldungssystem, das die Ergebnisse der eigenen Klinik vor dem Hintergrund vergleichbarer Suchtkliniken darstellt, schafft die Voraussetzung für eine Optimierung der Verlaufsprozesse. Sich aus dem Peer-Review ergebende Möglichkeiten wie auch mit dem Verfahren verbundene Probleme werden ausführlich diskutiert.

Schlüsselwörter: Qualitätssicherung, Abhängigkeitserkrankungen, Prozessqualität, Peer Review-Verfahren

Process Quality in the Inpatient Rehabilitation of Substance Dependency Patients: As part of the quality assurance program of the German state pension scheme's section for medical rehabilitation, a procedure for the evaluation of process quality was developed. This peer review procedure is tailored to the specific demands of substance dependency treatment. It utilizes the patients' medical discharge reports, which are drawn up using a standardized format as a source of information regarding the procedural quality of rehabilitation processes. The selected reports, chosen randomly from each clinic, are evaluated by experienced and trained specialists in the same field (peers) on the basis of both a detailed list of quality-related procedural characteristics and a manual. In an empirical evaluation, the peer review method,which has already been tested with regard to other rehabilitative

Suchttherapie 2000; 1: 126-136

(C) Georg Thieme Verlag Stuttgart · New York

ISSN 1439-9903
Stephan Kawski ${ }^{1}$, Ulrich Dorenburg ${ }^{2}$, Ulrike Beckmann ${ }^{3}$, Uwe Koch ${ }^{1}$

1 Universitätskrankenhaus Hamburg-Eppendorf, Abteilung für Medizinische Psychologie

2 Verband Deutscher Rentenversicherungsträger, Frankfurt/Main

${ }^{3}$ Bundesversicherungsanstalt für Angestellte, Berlin

indications, also sachieves satisfactory to good results in the field of substance dependency. The procedure allows for a differentiated description and analysis of the strengths and weaknesses of rehabilitative treatment measures. A detailed feedback system, permits performance data for individual clinics to be compared with data for other, similar against the dependency clinics, makes it possible to optimize the processes. Options for improving treatment which are offered by the peer review method of evaluation, as well as problems associated with this method, are discussed in detail.

Key words: Quality Assurance - Substance Dependency Process Quality - Peer-Review

\section{Das Qualitätssicherungsprogramm in der stationären Re-} habilitation der GRV

Das in seiner konzeptionellen Entwicklung stark durch die Rentenversicherung geprägte bundesdeutsche rehabilitative Versorgungssystem kann im internationalen Vergleich unter quantitativen und qualitativen Gesichtspunkten als sehr gut entwickelt betrachtet werden. Es ist geprägt von einem differenzierten und umfassenden Netzwerk rehabilitativer Versorgungseinrichtungen, deren Arbeit sich an den Ansätzen des Krankheitsfolgenmodells orientiert (vgl. ICIDH - International Classification of Impairments, Disabilities and Handicaps [1]). Hieraus begründet sich ein breites Spektrum von durchgeführten Behandlungen (somatisch, funktionsbezogen und psychosozial), welche in der Regel durch interdisziplinäre Behandlungsteams erbracht werden. Die medizinische Rehabilitation berücksichtigt dabei sozialmedizinische Erfordernisse und bindet Maßnahmen zur beruflichen und sozialen Wiedereingliederung ein. Mit diesem umfassenden Leistungsspektrum entwickelte sich das bundesdeutsche Rehabilitationssystem zu einem wichtigen Anbieter für die sekundäre bzw. tertiäre Prävention [2].

In den letzten Jahren wurde die öffentliche und politische Diskussion der Rehabilitation allerdings weniger durch die Stärken als durch eine Reihe kritischer Argumente bestimmt. Sie bezogen sich in einem Hauptpunkt auf ungeklärte Fragen der Nutzungsadäquanz und der Bedarfsorientierung. Zudem wurde der hohe Institutionalisierungsgrad der medizinischen Rehabilitation kritisiert, verbunden mit einer starken Betonung von stationären Leistungen und fehlenden ambulanten und wohnortnahen Maßnahmen sowie mit einer geringen Flexibilität der Leistungsangebote. Die Kritik einer unzurei- 
chenden Verzahnung richtete sich auf die Verbindung zwischen den verschiedenen (insbesondere den medizinischen und beruflichen) rehabilitativen Versorgungsfeldern, aber auch auf Schnittstellenprobleme zwischen kurativen, rehabilitativen und pflegerischen Maßnahmen. Weiter wurden Reibungsverluste durch die verschiedenen Träger- und Finanzierungszuständigkeiten konstatiert. Schließlich wurde nicht nur eine unzureichende wissenschaftliche Fundierung der Rehabilitation, sondern auch das Fehlen von Maßnahmen zur Überprüfung bzw. Weiterentwicklung der Qualität der Versorgung bemängelt.

Die Existenz von Strukturdefiziten wurde durch die sorgfältigen Analysen der vom Verband Deutscher Rentenversicherungsträger eingesetzten Kommission zur Weiterentwicklung der Rehabilitation in der Gesetzlichen Rentenversicherung [3] zumindest im Grundsatz bestätigt. In der Folge wurden deshalb seit Beginn der 90er Jahre eine größere Zahl von Veränderungsmaßnahmen initiiert. Die wesentlichen durch die Reformkommission veranlassten Innovationen betreffen vor allem Zielsetzungen wie stärkere Bedarfsorientierung, stärkere Orientierung an Qualitäts- und Ergebniskriterien, stärkere Flexibilisierung, Lösungen bei den Schnittstellenproblemen, Entwicklung neuer (nicht-stationärer) Angebotsformen sowie eine stärkere wissenschaftliche Orientierung der medizinischen Rehabilitation und eine Intensivierung der Qualitätssicherung.

Mit dem Ziel einer intensiveren Ergebnis- und Nutzerorientierung initiierte die Gesetzliche Rentenversicherung als eine der Reformmaßnahmen zu Beginn der 90er Jahre ein umfassendes Qualitätsmanagementprogramm. Seine Konzeption orientiert sich in wesentlichen Grundzügen an komplexen US-amerikanischen Qualitätssicherungsprogrammen aus anderen Versorgungsbereichen. Angelehnt an die Konzeption einer „kontinuierlichen Qualitätsverbesserung“ baut das Programm dabei auf das Prinzip dynamischer selbst lernender Systeme auf. Seine Verfahren ermöglichen eine Analyse wesentlicher Gesichtspunkte der Struktur-, Konzept-, Prozess- und Ergebnisqualität in den einzelnen Kliniken und eine ergebnisvergleichende Rückmeldung an die teilnehmenden Einrichtungen. ${ }^{1}$

Konzeptuell baut das Qualitätssicherungsprogramm auf der Problemanalyse innerhalb von vier Programmpunkten und einem Programmpunkt der Lösungserarbeitung auf:

- Programmpunkt 1 dient der Abbildung von „Strukturenund Konzepten“ der Einrichtungen und der Einteilung der Kliniken nach dem Prinzip ähnlicher Strukturen,

- im Programmpunkt 2 „Patiententherapiepläne“ sollen die Behandlungspläne für die Hauptbehandlungsgruppen der Kliniken nach prozessbezogenen Aspekten analysiert werden,

- zur Prozessqualität wird im Rahmen des Programmpunktes 3, des „Qualitäts-Screenings“, anhand konkreter Fälle die Sachgerechtheit in der Durchführung der rehabilitativen Maßnahme beurteilt (s.u.),

\footnotetext{
1 Darstellungen zur Entwicklung, Methodik und Umsetzung des 5-Punkte-Programms lassen sich u. a entnehmen bei Müller-Fahrnow [4], Schliehe [5], Egner et al. [6] und VDR [7].
}

- schließlich werden im Rahmen des Programmpunktes 4 Patientenbefragungen zur Zufriedenheit mit dem Aufenthalt und der Behandlung und zur Einschätzung des Therapieerfolges durchgeführt.

- Die Ergebnisse werden im Rahmen eines klinikvergleichenden Berichts- und Informationssystems zurückgemeldet. Die Rückmeldungen umfassen als einen wesentlichen Aspekt Hinweise auf Optimierungsbedarfe und bestehende Qualitätsmängel, hierzu sollen im Rahmen von angeleiteten problemorientierten Qualitätszirkeln (Programmpunkt 5) Lösungsmöglichkeiten erarbeitet werden.

Am Qualitätssicherungsprogramm der Rentenversicherung beteiligen sich alle 27 Rentenversicherungsträger sowie die Arbeitsgemeinschaft für Krebsbekämpfung in NordrheinWestfalen mit insgesamt 935 von ihnen federführend belegten Kliniken und Fachabteilungen. Die zentrale Koordination liegt beim VDR, der BfA und der LVA Westfalen. Die Verfahrens- und Methodenentwicklung erfolgte unter Beteiligung von vier externen wissenschaftlichen Instituten, unterstützt durch mehr als 100 Experten aus Klinik und Sozialmedizin.

\section{Das Peer Review-Verfahren als Zugang zur Prozessqualität}

Die im Qualitäts-Screening fokussierte objektive, verlässliche und valide Erfassung und Beurteilung der Prozessqualität in den Kliniken stellt eine besonders schwierige Aufgabe innerhalb eines Qualitätssicherungsprogramms dar. Bezogen auf den gewählten Zugang des Qualitäts-Screenings musste festgelegt werden, wodurch die Informationsgrundlage für die Evaluation der Behandlungsprozesse gebildet wird, nach welchen Kriterien und Grundlagen die Beurteilungen der Prozessqualität erfolgen sollen und wer die Qualität beurteilt.

Als Informationsgrundlage der Beurteilung wurden der einheitliche Entlassungsbericht der Rentenversicherung und die zugehörigen individuellen Therapiepläne gewählt. Sie liegen für jeden Patienten wenige Wochen nach der Durchführung der Rehabilitationsmaßnahme beim Rentenversicherungsträger vor. Dabei gewährleisten bundeseinheitlich bei allen Rentenversicherungsträgern geltende Richtlinien die Vergleichbarkeit. Für den Berichtsaufbau sind zunächst in weitgehend standardisierter Form eine Basisdokumentation wichtiger Patientendaten, die sozialmedizinische Leistungsbeurteilung und eine Dokumentation der therapeutischen Leistungsdaten vorgegeben. Der sich anschließende frei zu formulierende, aber an einem festen Gliederungsraster zu orientierende ärztliche Bericht geht regelmäßig auf die Aspekte „Allgemeine und klinische Anamnese“, „Jetzige Beschwerden und funktionelle Einschränkungen“, „Gegenwärtige Therapie und behandelnde Ärzte“, „Allgemeine Sozialanamnese“, „Berufsanamnese“, „Aufnahmebefund, Vorbefunde und ergänzende Diagnostik“, „Rehabilitationsdiagnosen und -ziele“, „Rehabilitationsverlauf“, „Rehabilitationsergebnis“ und „Sozialmedizinische Epikrise“ ein.

Die Beurteilung der Entlassungsberichte im Rahmen des Peer Reviews basiert auf einer mehrdimensionalen Checkliste. Diese beinhaltet qualitätsrelevante Prozessmerkmale, die gemeinsam mit ihren Indikatoren und den vorgesehenen Antwortkategorien durch ein Manual definiert und ausführlich unter Heranziehung von Beispielen erläutert werden. An 
der Erarbeitung der Checkliste qualitätsrelevanter Merkmale und des Manuals waren neben den Arbeitsgruppen der wissenschaftlichen Institute (s.u.) Vertreterinnen und Vertreter aus der rehabilitativen Praxis und der sozialmedizinischen Dienste beteiligt.

In Analogie zu wissenschaftlichen Beurteilungsprozessen im Rahmen von Publikationen und Anträgen auf Forschungsförderung sieht das Verfahren als Beurteiler den Einsatz erfahrener, unabhängiger und im Verfahren geschulter Fachkollegen des jeweiligen Indikationsbereiches („Peers“) vor. Dabei wird vorausgesetzt, dass die Beurteiler eine leitende Funktion in einer Rehabilitationseinrichtung wahrnehmen und mindestens über dreijährige praktische Erfahrung in diesem Arbeitsfeld verfügen. Außerdem wurde von ihnen erwartet, dass sie über die Zusatzbezeichnung „Sozialmedizin“ oder eine gleichwertige Qualifikation verfügen. Desweiteren mussten sie an einer Schulung für das Verfahren teilgenommen haben.

Das grundlegende Verfahrensmodell des Peer-Reviews für das Qualitäts-Screening der Rehabilitationsprozesse wurde von der Arbeitsgruppe um Prof. Jäckel (Hochrhein-Institut, Bad Säckingen) für die somatischen Rehabilitationsindikationen entwickelt [8].

Nachdem sich das Verfahren im Rahmen einer Pilotstudie mit 23 Rehabilitationskliniken als grundsätzlich praktikabel und erfolgversprechend erwiesen hatte, adaptierte die Forschungsgruppe Qualitätsmanagement der Abteilung für Medizinische Psychologie, Universitätskrankenhaus HamburgEppendorf, das Verfahren für die Indikationsbereiche Psychosomatik und Abhängigkeitserkrankungen. Hierzu war eine Reihe vor allem inhaltlicher Anpassungen der Checkliste qualitätsrelevanter Merkmale und des Manuals erforderlich. Die so entwickelte Variante des Verfahrens für die Indikationsbereiche Abhängigkeitserkrankungen und Psychosomatik wurde ebenfalls im Rahmen einer Pilotstudie geprüft, die auch hier die Praktikabilität und Objektivität des Verfahrens erwies.

In der sich an die Erprobung anschließenden ersten Phase der Routinisierung wurden ab 1997 zunächst 100 Kliniken aus den bedeutensten sechs somatischen Indikationsbereichen ${ }^{2}$ auf freiwilliger Basis in das Peer-Review einbezogen. Das hiermit beauftragte Hochrhein-Institut nahm neben der Schulung der Peers die grundlegende Prüfung der Praktikabilität (Bearbeitungsaufwand) des Verfahrens und dessen messtechnische Überprüfung bei der Übertragung auf die Versorgungspraxis vor. Das Ziel bestand darin, das Peer Review auf einer breiten Datengrundlage abzusichern und für einen effektiven Routineeinsatz zu optimieren. Weiterhin waren Prozeduren für die Ergebnisrückmeldung zu erarbeiten.

Aufbauend auf die positiven Ergebnisse dieses Arbeitsschrittes übernahm die Hamburger Forschungsgruppe Qualitätsmanagement ab Herbst 1998 den Auftrag, eine vergleichbare Überprüfung und Weiterentwicklung für die Indikationsbereiche Abhängigkeitserkrankungen und Psychosomatik zu

\footnotetext{
2 Orthopädie/Rheumatologie, Kardiologie, Onkologie, Neurologie, Pneumologie und Gastroenterologie
}

leisten. An den Schulungen und Beurteilungen nahmen im Jahre 199921 Kliniken aus dem Bereich Psychosomatik und 30 Kliniken für die Behandlung von Abhängigkeitserkrankungen teil. Die unten berichteten Ergebnisse beziehen sich auf die Peer-Reviews in den 30 beteiligten Einrichtungen aus dem Indikationsbereich Abhängigkeitserkrankungen.

\section{Das Peer Review im Bereich Abhängigkeitserkrankungen}

Der Ergebnisdarstellung ist eine Beschreibung des Verfahrens in seiner Anpassung an die Besonderheiten in der Suchtrehabilitation vorangestellt. Weiterhin werden die Verfahrensabläufe und die Rückmeldeprozedur erläutert.

\section{Checkliste qualitätsrelevanter Prozessmerkmale und Manual}

Die für die Indikationsbereiche Abhängigkeitserkrankungen und Psychosomatik adaptierte Checkliste umfasst insgesamt 67 qualitätsrelevante Prozessmerkmale. Sie gliedert sich in die Bereiche Anamnese, Diagnostik, Therapieziele und -planung, Verlauf und Epikrise, Sozialmedizinische Stellungnahme und weiterführende therapeutische Maßnahmen (vgl. Tab. 1). Für die Bewertung der einzelnen Prozessmerkmale stehen die drei Kategorien „keine Mängel“, „leichte Mängel“ und „gravierende Mängel“ zur Verfügung sowie eine zusätzliche Kategorie „entfällt“. Für jeden der genannten Bereiche findet zusätzlich eine zusammenfassende Beurteilung statt, für die vier Kategorien zur Verfügung stehen („keine Mängel“, „leichte Mängel“, „deutliche Mängel“ und „gravierende Mängel“). Ebenfalls anhand dieser vier Beurteilungskategorien erfolgt zum Abschluss der Begutachtung eine zusammenfassende Bewertung des gesamten Reha-Prozesses. Damit liegt für jede in das Verfahren aufgenommene Dokumentation eine Bewertung zu 67 Einzelmerkmalen, sechs übergeordneten Bereichen und zum Gesamtprozesses vor.

Die Einzelkriterien und die Bereiche der Checkliste werden im zugehörigen Manual definiert und operationalisiert. Das Manual enthält für jedes Prozessmerkmal Kriterien, die der Qualitätsbeurteilung zugrunde gelegt werden müssen. Abb. 1 zeigt beispielhaft für das Prozessmerkmal A 1.3 „Bisherige diagnostische Maßnahmen“ den Aufbau des Manuals.

Prinzipiell muss sich die Beurteilung eines qualitätsrelevanten Prozessmerkmals an den im Manual aufgeführten Kriterien orientieren. Die zusammenfassenden Bewertungen von Bereichen müssen sich entsprechend an den zuvor vorgenommenen Beurteilungen der entsprechenden qualitätsrelevanten Prozessmerkmale ausrichten. Dabei sollen bei der Beurteilung der einzelnen qualitätsrelevanten Prozessmerkmale und bei den zusammenfassenden Bewertungen sowohl quantitative (Anzahl erfüllter Kriterien bzw. Merkmale) als auch qualitative Gesichtspunkte (Gewichtung einzelner Kriterien bzw. Merkmale nach den spezifischen Gegebenheiten des jeweiligen Falls) zugrunde gelegt werden. Bei den zusammenfassenden Bewertungen ist eine integrierte Bewertung erforderlich, die berücksichtigt, welchen Stellenwert gegebenenfalls festgestellte Mängel für eine fachgerechte Durchführung der gesamten Reha-Maßnahme in dem vorliegenden Fall hatten. 


\begin{tabular}{ll}
\hline Gliederungspunkt & Beispiel \\
\hline $\begin{array}{l}\text { Qualitätsrelevantes } \\
\text { Prozessmerkmal } \\
\text { Indikatoren / Kriterien }\end{array}$ & A.1.3. Bisherige diagnostische Maßnahmen \\
& - bisherige somatische, funktionelle und \\
psychosomatische Diagnostik & Befunde aus Vorgutachten \\
- ggf. Hinweis, dass keine früheren & diagnostischen Maßnahmen erfolgten \\
Beispielhafte Anwendung & $\begin{array}{l}\text { Ein Entlassungsbericht weist hier gravierende Mängel auf, wenn } \\
\text { keinerlei Informationen mitgeteilt werden, ob frühere } \\
\text { diagnostische Maßnahmen erfolgten, und wenn ja, welche. } \\
\text { Aufgrund der Einweisungsdiagnosen ist i.d.R. davon } \\
\text { auszugehen, dass frühere diagnostische Maßnahmen } \\
\text { unternommen wurden. }\end{array}$ \\
\hline
\end{tabular}

Abb. 1 Struktur des Manuals zur Checkliste qualitätsrelevanter Prozessmerkmale.

\section{Verfahrensablauf}

Die Grundlage für die Einschätzung der Prozessqualität in einer Einrichtung bildet eine durch den Rentenversicherungsträger zufallsausgewählte Stichprobe von individuellen Entlassungsberichten. Um eine Vergleichbarkeit der Ergebnisse zu gewährleisten, wird i.d.R. ein sechsmonatiger Zeitraum festgelegt, in dem die zugrunde gelegten Behandlungen abgeschlossen worden sein müssen. Dabei liegt dieser Zeitraum möglichst kurze Zeit zurück, um eine Aktualität der Beurteilungen $\mathrm{zu}$ sichern.

Die Anonymisierung der einzusendenden Berichte, insbesondere bezogen auf schutzwürdige Daten der Patienten, erfolgt bereits in der Klinik. Anschließend werden die Berichte vor Weitergabe an die Auswertungsstellen beim VDR und der BfA durch den zuständigen Rentenversicherungsträger dahingehend anonymisiert, dass die einzelne Klinik nur noch über eine Code-Nummer identifizierbar ist.

Auch die Verteilung der Berichte auf die begutachtenden Peers geschieht zufallsgestützt. Abgesichert wird hierbei jedoch, dass ein Peer nicht Berichte seiner eigenen Einrichtung zur Bewertung erhält. Die Berichte einer Klinik werden grundsätzlich auf die größtmögliche Anzahl verschiedener Peer verteilt.

Die Peers schicken nach durchgeführter Begutachtung die Berichte gemeinsam mit den bearbeiteten Checklisten an die Auswertungsstellen zurück, bei denen anschließend die Dateneingabe und -kontrolle erfolgen. Auf der Basis des so erstellten Datensatzes erfolgt nun die Datenauswertung und die Erstellung von Rückmeldeberichten durch das beteiligte wissenschaftliche Institut (Abteilung für Medizinische Psychologie, Universitätskrankenhaus Hamburg-Eppendorf). Nach Prüfung der Arbeitsergebnisse durch die Auswertungsstellen werden die klinikbezogenen Berichte über die zuständigen Rentenversicherungsträger an die beteiligten Einrichtungen rückgemeldet.

\section{Klinikbezogener Ergebnisbericht}

Für jede teilnehmende Klinik wird ein Ergebnisbericht erstellt, der neben einer methodischen Einführung eine detaillierte Rückmeldung der Beurteilungen durch die Peers hinsichtlich der beschriebenen Bewertungsdimensionen umfasst. Daraus kann ersehen werden, in welchen Prozessbereichen bzw. bei welchen qualitätsrelevanten Merkmalen Probleme oder Stärken bestehen. Dabei erhalten die Kliniken ergänzend Informationen über ihre Position im Vergleich zu den anderen Kliniken ihres Indikationsbereichs bei den zusammenfassenden Bewertungen zu den sechs Beurteilungsbereichen und zum Gesamtprozess.

Der Ergebnisteil der Rückmeldungen an die Kliniken gliedert sich in:

- grafische Darstellungen der zusammenfassenden Bewertungen des gesamten Reha-Prozesses und der einzelnen Bereiche der Checkliste mit Gegenüberstellungen der Bewertungen der Klinik mit den durchschnittlichen Verteilungen für alle Kliniken des Indikationsbereichs und für das beste und das schlechteste Ergebnis,

- eine tabellarische Übersicht $\mathrm{zu}$ den grafischen Darstellungen mit den individuellen Klinikwerten und den Verteilungswerten aller Kliniken des Indikationsbereiches,

- eine entsprechende Tabelle der Bewertungen der Einzelmerkmale der Checkliste, in der zusätzlich Unterschiede zwischen Werten der jeweiligen Einrichtung und den Durchschnittswerten aller Einrichtungen des Indikationsbereichs auch auf statistische Signifikanz getestet werden, und

- ein Polaritätenprofil, das einen prägnanten Überblick über besondere Stärken und Schwächen der jeweiligen Klinik in den Einzelmerkmalen vor dem Hintergrund der Ergebnisse der anderen Kliniken des Indikationsbereichs bietet. Hierfür wurde für jede Klinik ermittelt, welche Einzelmerkmale deutlich besser oder deutlich schlechter beurteilt wurden als in der Gesamtgruppe der Kliniken des betreffenden Indikationsbereichs.

Mit diesem Aufbau orientiert sich die Ergebnisrückmeldung am Entscheidungs- und Bearbeitungsvorgehen der Qualitätszirkel in den Reha-Kliniken. Globale Hinweise auf Schwachstellen und mögliche Optimierungsbedarfe lassen sich den grafischen Vergleichen der Verteilungen und den Polaritätenprofilen entnehmen. Weitergehende Analysen, Entscheidungen und Verbesserungsansätze können auf den differenzierten tabellarischen Mitteilungen aufbauen, bei denen die Ergebnisse der Signifikanztests zusätzliche Unterstützung für die Ergebnisinterpretation anbieten. 


\section{Empirische Erprobung des Peer Review-Verfahrens für den Indikationsbereich Abhängigkeitserkrankungen}

\section{Fragestellung und methodischer Zugang}

Ab Herbst 1998 wurde das Peer Review-Verfahren für den Indikationsbereich Abhängigkeitserkrankungen im Rahmen einer ersten Stufe der Routinisierung in 30 freiwillig teilnehmenden Einrichtungen eingeführt. Unter methodischen Gesichtspunkten sollte dabei geprüft werden, ob das Verfahren für den Routineeinsatz hinreichend praktikabel ist, ob eine ausreichende Interrater-Reliabilität erreicht wird (mit einer Abschätzung eventuell notwendiger Kontroll- und KorrekturStrategien) und ob eine Differenzierung zwischen den Ergebnissen der beteiligten Einrichtungen erreicht werden kann.
Die eingesetzten Begutachter („Peers“) wurden zuvor von den teilnehmenden Kliniken benannt und erfüllten die im Abschnitt 2 als Grundvoraussetzungen zur Verfahrensteilnahme beschriebenen Kriterien. Sie wurden vor der Durchführung der Beurteilungen im Rahmen von zweitägigen Trainings in das Verfahren eingeführt und im Gebrauch der Checkliste und des Manuals geschult.

Aus jeder der 30 an der Prüfung beteiligten Einrichtungen für Abhängigkeitserkrankungen wurden grundsätzlich 25 Entlassungsberichte (Entlassungszeitraum in der Regel Mai bis Oktober 1998) zufallsgestützt bestimmt, die in das PeerReview-Verfahren eingingen. Nach Durchlaufen der mehrstufigen Anonymisierungs- und Bewertungsprozedur standen nach Datenkontrolle und -korrektur für die statistischen

Tab. 1 Bereiche und qualitätsrelevante Prozessmerkmale der Checkliste

Bereich (A): Anamnese

A. 1 Spezielle, auf die Hauptindikation bezogene Anamnese:

A.1.1 Aktuelle Beschwerden

A.1.2 Krankheitsverlauf

A.1.3 Bisherige diagnostische Maßnahmen

A.1.4 Bisherige therapeutische Maßnahmen

A.1.5 Funktionseinschränkungen im Alltag

A.1.6 Funktionseinschränkungen im Beruf

A.1.7 Krankheitsverständnis

A.1.8 Zuweisungsdiagnose genannt

A.2 Weitere reha-relevante psychische u. somatische Erkrankungen:

A.2.1 Vorerkrankungen

A.2.2 Ko- und Multimorbidität (mit Ang. zu A.1.1-A.1.6)

A.3 Krankheitsrelevante biografische Anamnese

A.4 Krankheitsrelevante Familienanamnese

A. 5 Vegetative Beschwerden

A.6 Derzeitige Risikofaktoren und Risikoverhalten:

A.6. 1 Somatisch

A.6.2 Psychosozial

A.7 Derzeitige Belastungsfaktoren

A.8 Sozialmedizinische Anamnese:

A.8.1 Beruflicher Werdegang

A.8.2 Beschreibung der letzten versicherungspflichtigen Tätigkeit

A.8.3 Erwerbsstatus

A.8.4 AU-Zeiten

A.9 Initiative zur Reha-Antragstellung:

A 9.1 Behandlungsmotivation

A 9.2 Reha-Vorbereitung und Zugang

Bereich (B): Diagnostik

B. 1 Medizinische Untersuchungen und Befunde:

B.1.1 Allgemeinärztlicher Befund

B. 1.2 Indikationsspezifischer internistischer Befund

B.1.3 Technische Befunde

B. 1.4 Zusätzliche Befunde aus anderen Fachgebieten

B.2 Psychiatrische u. psychologische Untersuchungen/Befunde:

B.2.1 Psychischer bzw. psychopathologischer Befund

B.2.2 Psychodynamischer Befund

B.2.3 Verhaltensanalytischer Befund

B.2.4 Testpsychologische Befunde

B.2.5 Weitere psychosoziale und interaktionelle Diagnostik

B.2.6 Verhaltensaktiva

B.3 Indikationsbezogene Fähigkeits-/Funktionsstörungen

B.4 Zusammenschau/Aggregation der Diagnostik
Bereich (C): Therapieziele und -planung

C. 1 Ziele adäquat für Reha-Problem des Patienten

C.2 Ziele konkret formuliert

C.3 Ziele mit Patienten besprochen

C.4 Individueller Behandlungsplan schlüssig dargestellt

C.5 Behandlungsprogramm adäquat für Reha-Problem:

C.5. 1 Art der Behandlungsmaßnahmen adäquat

C.5.2 Häufigkeit angemessen

C.5.3 Reihenfolge u. Verteilung sinnvoll

C.6 Gesundheitsinformation/-training adäquat

Bereich (D): Verlauf und Epikrise

D. 1 Diagnosen durch Anamnese und Befund plausibel

D.2 Änderung der Zuweisungsdiagnose nötig

D.3 Darstellung des Reha-Verlaufs:

D.3.1 Medizinischer Teil

D.3.2 Psychotherapeutischer Teil

D.3.3 Sozialtherapeutischer und berufsbezogener Teil

D.4 Veränderung der Reha-Dauer begründet

D.5 Motivation und Kooperation des Patienten

D.6 Behandlungsergebnis / Abschlussbefund:

D.6. 1 Therapeuteneinschätzung

D.6.2 Patientenselbsteinschätzung

D.7 Aussagen zur Übereinstimmung von Beschwerden und erhobenen Befunden

D.8 Kritische Würdigung: Reha-Prozess u. erreichte Ergebnisse:

D.8.1 Medizinischer Teil

D.8.2 Psychotherapeutischer Teil

D.8.3 Sozialtherapeutischer und berufsbezogener Teil

Bereich (E): Sozialmedizinische Stellungnahme

E. 1 Bezogen auf die letzte berufliche Tätigkeit:

E.1.1 Qualitative Beurteilung

E.1.2 Quantitative Beurteilung

E.2 Bezogen auf das positive und negative Leistungsbild: E.2.1 Qualitative Beurteilung

E.2.2 Quantitative Beurteilung

E.3 Empfehlungen für berufsfördernde Maßnahmen

E.4 Selbsteinschätzung des Patienten zur beruflichen Leistungsfähigkeit

E.5 Bezogen auf die Selbstversorgung/Pflegebedürftigkeit

Bereich (F): Weiterführende Maßnahmen/Nachsorge

F.1 Empfehlungen plausibel u. adäquat:

F.1.1 Weitere Diagnostik

F.1.2 Weitere Therapie:

F.1.2.1 Medizinische Therapie

F.1.2.2 Psychosoziale Therapie

F.2 Konkrete Einleitung von weiteren (ambulanten Reha-)Maßnahmen einschließlich Nachsorge

F.3 Eigene Pläne des Patienten 


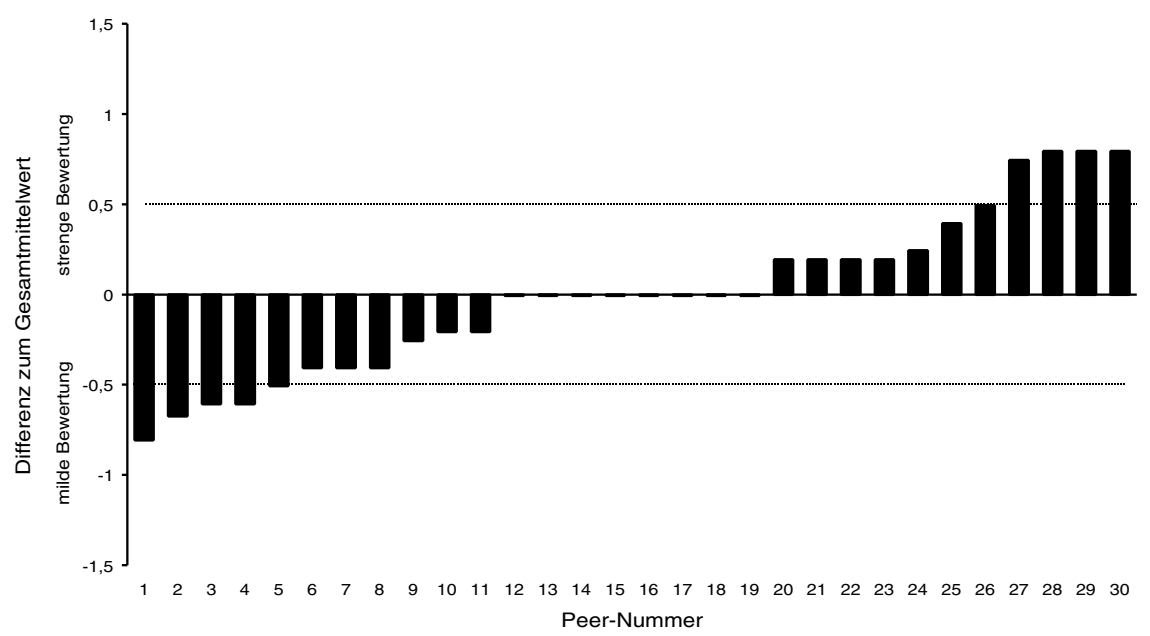

Abb. 2 Systematische Beurteilerunterschiede: Zusammenfassende Bewertungen des Reha-Prozesses; gekennzeichnet ist der Bereich von \pm 0,5 Punkten Abweichung (70\% der Peers).

Analysen die Bewertungen von 604 Entlassungsberichten als Beurteilungsstichprobe zur Verfügung - durchschnittlich etwa 20 bewertete Berichte pro Klinik. ${ }^{3}$

Über die Beurteilungsstichprobe hinaus wurden zusätzlich fünf Kontrollberichte ausgewählt, die von allen Peers des Indikationsbereichs bewertet wurden. Sie bildeten die Grundlage für die Bestimmung des Grades der Übereinstimmung zwischen den Peers.

Methodische Details der Auswertungsschritte werden nachfolgend in den jeweiligen Abschnitten mitgeteilt.

\section{Ergebnisse der testtheoretischen Überprüfung des Verfahrens}

\section{Bearbeitungsdauer}

Da das Peer Review auf eine Beurteilung von Prozessdokumentationen durch leitende Kliniker aufbaut, ist es erforderlich, den zeitlichen Aufwand für die Bewertungen in einem angemessenen Verhältnis zum angestrebten Ergebnis zu halten. Insofern bezog sich die erste Prüffragestellung auf die Ökonomie der Durchführung des Verfahrens.

Auf der Basis der Selbstangaben der Peers ergab sich eine Bearbeitungsdauer von durchschnittlich 33,4 Minuten. In $88 \%$ der Fälle waren für die Bearbeitung eines Berichts nicht mehr als 45 Minuten nötig, für 2,5\% der Begutachtungen wurden mehr als 60 Minuten aufgewendet. Da durch jeden Peer maximal 25 Entlassungsberichte (einschließlich Kontrollberichte) zu bewerten sind, beträgt der zeitliche Aufwand eines Peers bei einem Durchgang des Verfahrens durchschnittlich etwa 13 Stunden. Dieser Zeitaufwand wird derzeit als angemessen eingeschätzt, wenn man bedenkt, dass die teilnehmenden Kliniken im Gegenzug aggregierte Rückmeldungen über wichtige Aspekte der Prozessqualität erhalten auf der Basis von Beurteilungen durch eine Gruppe von erfahrenen und besonders qualifizierten Reviewern. Im wei-

\footnotetext{
${ }^{3}$ Allerdings reduzierte sich dieser Umfang bei einzelnen der in den nachfolgenden Abschnitten dargestellten Analysen aufgrund fehlender Angaben in begrenztem Ausmaß.
}

teren Fortlauf des Qualitäts-Screenings sollte dennoch geprüft werden, durch welche Modifizierungen des Verfahrens und Vorgehens eine Entlastung für die Beurteiler erreicht werden kann.

\section{Interrater-Reliabilität}

Als ein weiteres Testgütemaß wurde der Grad der Übereinstimmung zwischen den Peers (Interrater-Reliabilität) überprüft. Die Grundlage hierfür bildeten je Indikationsbereich fünf so genannte Kontrollberichte, die von jedem Peer zu beurteilen waren. Damit bezogen sich für den Indikationsbereich Abhängigkeitserkrankungen 145 Kontrollbewertungen auf die 5 Berichte $^{4}$.

Die Interrater-Reliabilität wurde nach Finn $[9,10]$ ermittelt. Diese Methodik wurde im Rahmen des Peer-Review-Verfahrens gewählt, da sie bei geringer Varianz zwischen den Entlassungsberichten hinsichtlich der Bereiche und der zusammenfassenden Bewertung ein robustes Äquivalent zur sonst üblichen Ermittlung der Intraklassenkorrelation darstellt [11].

Der Koeffizient beträgt für die zusammenfassende Bewertung des gesamten Reha-Prozesses für Abhängigkeitserkrankungen wie für die zusammenfassenden Bewertungen der einzelnen Bereiche der Checkliste im Indikationsbereich Abhängigkeit zwischen 0,5 und 0,64 (vgl. Tab. 2). Damit ist die Gutachterübereinstimmung für die zusammenfassenden Bewertungen des Reha-Prozesses und der einzelnen Bereiche im Indikationsbereich Abhängigkeitserkrankungen als zufriedenstellend einzustufen.

\footnotetext{
4 Die Stichprobe der Kontrollberichte wurde zufällig aus der Gesamtgruppe der Entlassungsberichte gezogen. Die Peers hatten in der Regel jeweils fünf Kontrollberichte zu beurteilen; stammte ein zu bewertender Kontrollbericht aus der eigenen Klinik eines Peers, war er entsprechend den Regeln des Gesamtverfahrens nicht zu bearbeiten; in diesem Fall bewertete der Peer nur vier Kontrollberichte.
} 
Tab. 2 Interrater-Reliabilitäten für die zusammenfassenden Bewertungen der einzelnen Bereiche der Checkliste und die zusammenfassende Bewertung des gesamten Reha-Prozesses (Grundlage: 5 Berichte, bearbeitet von 30 Peers)

Bewertungsgrundlagen

Interrater-Reliabilitäten (Finn-Koeffizienten)

(A) Anamnese

(B) Diagnostik

0,61

0,50

(D) Verlauf und Epikrise

(E) sozialmedizinische Stellungnahme

(F) weiterführende therapeutische Maßnahmen

zusammenfassende Bewertung

\section{0,60}

0,54

0,54

0,64

0,53

\section{Unterschiede der Beurteilerstrenge}

Bei einem solchen Begutachtungsverfahren ist es prinzipiell möglich, dass einige Gutachter generell zu „strengen“ oder „milden“ Bewertungen neigen. Für den Fall gravierender systematischer Unterschiede im Bewertungsverhalten wäre mit unterschiedlichen Strategien vorzugehen. So könnte es erforderlich sein, Gewichtungen vorzunehmen oder gegebenenfalls Gutachter, die in ihrer Beurteilung erheblich vom Durchschnitt abweichen, von dem Verfahren auszuschließen.

In den Analysen anhand der Kontroll-Entlassberichte, die durch alle Peers begutachtet wurden, ließen sich systematische Beurteilerunterschiede finden. Diese streuen aber in einem engen Bereich um den Mittelwert aller Bewertungen. Insgesamt zeigt sich ein gut vergleichbares Ausgangsniveau in Bezug auf die Bewertungsstrenge/-milde. Von 70\% der Peers weichen die Bewertungen durchschnittlich um weniger als 0,5 Bewertungsstufen vom Mittelwert aller Peers des Indikationsbereichs ab - selbst die höchste durchschnittliche Abweichung beträgt lediglich 0,8 Bewertungsstufen (vgl. Abb. 2). Dennoch sollte bei der Interpretation der nachfolgend dargestellten Ergebnisse zur Prozessbeurteilung berücksichtigt werden, dass die Konfidenzbereiche für jeden einzelnen
Peer im Indikationsbereich Abhängigkeitserkrankungen durchschnittlich bis zu 0,28 Bewertungsstufen betragen.

Individuelle Mittelwertunterschiede zwischen den Beurteilern - wenngleich quantitativ begrenzt - begründen die Frage, wie sich die vorgenommene Verteilung der Berichte auf die Gesamtbewertungen der einzelnen Kliniken im PeerReview-Verfahren auswirkt. Theoretisch wäre es denkbar, dass die Entlassungsberichte aus einer Klinik überdurchschnittlich häufig von „strengeren“ Peers bzw. „milderen“ Peers beurteilt würden. Diese Klinik würde dann aufgrund der systematischen Unterschiede im Beurteilungsverhalten der Peers entsprechend schlecht bzw. gut abschneiden.

Im Peer Review-Verfahren wird versucht, diesem Risiko zu begegnen, indem die Entlassungsberichte einer Einrichtung randomisiert auf die größtmögliche Anzahl verschiedener Beurteiler verteilt werden. Im Mittel verteilten sich in der durchgeführten Erprobung die Entlassungsberichte einer Klinik auf 17 Peers. Damit wurde die gewünschte Streuung der Berichte über die Peers durch das Zuweisungsverfahren erreicht.

Ob damit auf eine Korrektur von individueller Beurteilerstrenge (oder -milde) verzichtet werden kann, wurde geprüft, indem für jede Klinik ein Vergleich der mittleren „zusammenfassenden Bewertung des gesamten Reha-Prozesses“ mit und ohne einen Korrekturfaktor erfolgte, der die unterschiedlichen Mittelwerte der einzelnen Beurteiler berücksichtigt. Die Ergebnisse zeigen für die 30 Kliniken des Indikationsbereichs Abhängigkeitserkrankungen nur marginale Unterschiede zwischen den korrigierten und unkorrigierten Klinikmittelwerten (vgl. Abb. 3).

Zusammenfassend ist damit belegt, dass das Randomisierungsverfahren systematische Unterschiede zwischen den Peers weitestgehend ausbalanciert und deshalb sowohl auf das Herausnehmen einzelner, besonders „strenger“ oder „milder“, Beurteiler als auch auf die Einführung einer beurteilerbezogenen Abweichungskorrektur verzichtet werden kann.

\section{Effekte der Abweichungskorrektur Abhängigkeitserkrankungen}

Abb. 3 Klinikmittelwerte (Zusammenfassende Bewertung des Reha-Prozesses) mit/ ohne Korrektur.

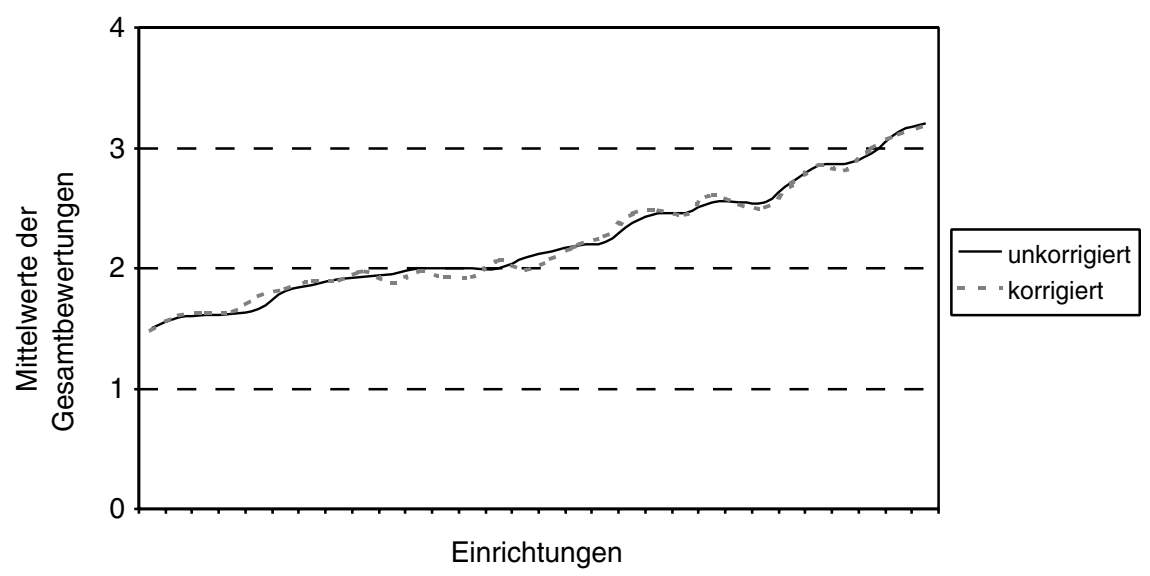




\begin{tabular}{lllll}
\hline Bereich & $\begin{array}{l}\text { keine } \\
\text { Mängel }\end{array}$ & $\begin{array}{l}\text { leichte } \\
\text { Mängel }\end{array}$ & $\begin{array}{l}\text { deutliche } \\
\text { Mängel }\end{array}$ & $\begin{array}{l}\text { gravierende } \\
\text { Mängel }\end{array}$ \\
\hline Anamnese & 29,3 & 47,2 & 18,4 & 5,2 \\
Diagnostik & 23,0 & 43,9 & 25,9 & 7,2 \\
Therapieziele und -planung & 31,9 & 38,9 & 20,0 & 9,2 \\
Verlauf und Epikrise & 35,7 & 40,8 & 16,3 & 7,2 \\
sozialmedizinische Stellungnahme & 41,3 & 32,9 & 17,1 & 8,7 \\
weiterführende Maßnahmen/Nachsorge & 53,3 & 31,4 & 11,4 & 4,0 \\
zusammenfassende Bewertung & 19,8 & 46,6 & 25,6 & 8,0 \\
\hline
\end{tabular}

Tab. 3 Zusammenfassende Bewertungen für die einzelnen Bereiche des Reha-Prozesses und zusammenfassende Bewertung des gesamten Prozesses (Angaben in \%) (Grundlage: 604 Fallberichte aus 30 Kliniken für Suchtrehabilitation)

\section{Differenzierungsmöglichkeiten der Einrichtungen nach dem Grad der Prozessqualität}

Eine zentrale Anforderung an das für das Qualitäts-Screening entwickelte Verfahren besteht in der Eignung zur Differenzierung der Ergebnisse der einzelnen Einrichtungen. Für Entscheidungen über Qualitätsverbesserungsansätze werden in den Einrichtungen Ergebnisrückmeldungen benötigt, die auch Vergleiche zu den anderen Einrichtungen des Indikationsbereiches umfassen. Hierbei sind grundsätzlich zwei Zugänge denkbar: eine Rangreihung der Einrichtungen nach ihren Ergebnissen im Sinne eines Benchmarkings und ein Vergleich der Ergebnisse der einzelnen Einrichtungen mit denen der Gesamtgruppe.
Für die zusammenfassenden Bewertungen der sechs Bereiche und der Gesamtprozesse lassen sich aufgrund weiter Konfidenzintervalle die Mittelwerte der einzelnen Einrichtungen nur mit erheblicher Unsicherheit für ein Benchmarking zugrunde legen. Deshalb wurde, um vergleichende Rückmeldungen zu geben, eine Gegenüberstellung der relativen Häufigkeiten der Beurteilungen vorgenommen.

Für die auf Einzelitems bezogene Darstellung spezifischer Stärken und Schwächen ist das Verfahren gut geeignet, die relativen Häufigkeiten der Bewertungen für Einzelkliniken den Verteilungen für alle Einrichtungen des Indikationsbereiches gegenüberzustellen und (non-parametrisch) zu vergleichen. Eine Aufdeckung von besonderen Stärken und Schwachstellen der Einzeleinrichtungen, im Vergleich zu allen Einrichtungen des Indikationsbereiches, wird weiterhin

\begin{tabular}{|c|c|c|}
\hline Bereich & Merkmal & $\begin{array}{c}\text { gravierende } \\
\text { Mängel (\%) }\end{array}$ \\
\hline Anamnese & $\begin{array}{l}\text { Funktionseinschränkungen im Beruf } \\
\text { subjektives Krankheitsverständnis } \\
\text { vegetative Beschwerden } \\
\text { letzte berufliche Tätigkeit } \\
\text { Vorbereitung der Reha-Behandlung }\end{array}$ & $\begin{array}{l}12,6 \\
12,5 \\
15,6 \\
12,6 \\
13,0\end{array}$ \\
\hline Diagnostik & $\begin{array}{l}\text { psychodynamischer Befund } \\
\text { verhaltensanalytischer Befund } \\
\text { testpsychologischer Befund } \\
\text { Verhaltensaktiva } \\
\text { Aggregation der Diagnostik }\end{array}$ & $\begin{array}{l}25,5 \\
17,9 \\
19,8 \\
17,7 \\
16,3\end{array}$ \\
\hline $\begin{array}{l}\text { Therapieziele und } \\
\text {-planung }\end{array}$ & $\begin{array}{l}\text { Ziele konkret formuliert } \\
\text { Ziele mit Patienten besprochen } \\
\text { individuelles Behandlungskonzept schlüssig } \\
\text { angemessene Reihenfolge der Maßnahmen } \\
\text { Gesundheitsinformation adäquat }\end{array}$ & $\begin{array}{l}13,4 \\
24,7 \\
23,9 \\
13,8 \\
20,6\end{array}$ \\
\hline Verlauf und Epikrise & $\begin{array}{l}\text { Ergebniseinschätzung durch den Patienten } \\
\text { Verlauf: sozialtherapeutisch/berufsbezogener Teil } \\
\text { Verlauf: medizinischer Teil } \\
\text { kritische Würdigung: sozialtherapeutischer Teil } \\
\text { kritische Würdigung: medizinischer Teil }\end{array}$ & $\begin{array}{l}17,9 \\
11,0 \\
10,2 \\
12,4 \\
10,4\end{array}$ \\
\hline $\begin{array}{l}\text { sozialmedizinische } \\
\text { Stellungnahme }\end{array}$ & $\begin{array}{l}\text { Selbsteinschätzung des Patienten } \\
\text { qualitative Leistungsbild-Beurteilung (nach Abschluss) } \\
\text { quantitative Leistungsbild-Beurteilung (nach Abschluss) } \\
\text { qualitative Beurteilung (letzte Tätigkeit berücksichtigt) } \\
\text { Empfehlungen für berufsfördernde Maßnahmen }\end{array}$ & $\begin{array}{l}27,8 \\
16,4 \\
10,5 \\
14,4 \\
13,4\end{array}$ \\
\hline $\begin{array}{l}\text { weiterführende } \\
\text { therapeutische } \\
\text { Maßnahmen / } \\
\text { Nachsorge }\end{array}$ & $\begin{array}{l}\text { eigene Pläne des Patienten } \\
\text { Einleitung weiterer Maßnahmen } \\
\text { Diagnostikempfehlungen plausibel } \\
\text { medizinische Therapieempfehlungen plausibel } \\
\text { psychosoziale Therapieempfehlungen plausibel }\end{array}$ & $\begin{array}{l}9,6 \\
9,5 \\
7,9 \\
7,7 \\
7,4\end{array}$ \\
\hline
\end{tabular}

Tab.4 Qualitätsrelevante Prozessmerkmale mit vergleichsweise hohen Anteilen an problematischen Einschätzungen (Grundlage: 604 Fallberichte aus 30 Kliniken für Suchtrehabilitation) 
möglich, indem Prozessmerkmale ausgewählt werden, die für die Einzeleinrichtung vom jeweiligen Mittelwert für alle Einrichtungen des Indikationsbereiches um mehr als eine Standardabweichung (der Gesamtgruppe) abweichen.

Insgesamt zeigten die Erprobungen hier, dass eine Differenzierung der Ergebnisse der Einzelkliniken von der Gesamtgruppe der Einrichtungen auf diesem Wege erreicht werden kann. Statistische Absicherungen sind aber vor dem Hintergrund der pro Einrichtung vergleichsweise kleinen Stichproben auch mit Unsicherheiten belastet.

\section{Analyse der Prozessqualität}

Das Ziel des Peer Reviews ist es, wie zuvor beschrieben, den Klinikern eine Rückmeldung über die Prozessqualität in der eigenen Einrichtung im Vergleich zu anderen Einrichtungen zu geben. Betrachtet man die Ergebnisse aller Kliniken, so ergibt die Auswertung gleichzeitig einen Eindruck über die gegenwärtigen Stärken und Schwächen innerhalb des Versorgungsbereichs Suchtrehabilitation und über eventuelle Optimierungsbedarfe. Mit dieser Zielsetzung werden in den nachfolgenden Darstellungen für die sechs übergeordneten Bereiche der Checkliste die jeweiligen kategorialen Häufigkeiten der Beurteilungen (\%) mitgeteilt. Für die 67 Einzelmerkmale werden lediglich die Prozentsätze der gravierenden Mängel angegeben.

Die Berechnungen basieren auf 604 Checklisten. Datenausfälle reduzieren die Stichproben jeweils in geringem Umfang.

\section{Prozessqualität auf der Ebene der zusammenfassenden Bewertungen}

In Tab. 3 sind die prozentualen Verteilungen der zusammenfassenden Bewertungen dargestellt - zunächst der einzelnen Bereiche und anschließend des gesamten Reha-Prozesses. (Vergleiche mit anderen Indikationsbereichen sollten auf dieser Basis allerdings nicht vorgenommen werden, da hier unterschiedliche Reha-Prozesse den Bewertungen unterzogen werden.)

Betrachtet man die Beurteilungen „keine Mängel“ und „leichte Mängel“ insgesamt als positive Einschätzungen, so zeigt sich auf der Basis von mehr als 600 beurteilten Prozessdokumentationen ein Anteil günstiger Einschätzungen je nach Bereich von bis zu 85 Prozent. Fasst man die Bewertungen „deutliche Mängel“ und „gravierende Mängel“ zusammen, dann liegen die Werte für die Bereiche Diagnostik $(33,1 \%)$, Therapieziele und -planung $(29,2 \%)$ sowie sozialmedizinische Stellungnahme $(25,8 \%)$ am höchsten und weisen auf einen deutlichen Optimierungsbedarf hin. Für die zusammenfassenden Bewertungen der gesamten Reha-Prozesse ergibt sich ein Anteil von insgesamt 33,6\% deutlicher und gravierender Mängel. Zusammenfassend ist festzustellen, dass je betrachteter Fragestellung der Anteil der als substanziell eingeschätzten Mängel zwischen etwa $1 / 3$ und etwa $1 / 7$ liegt. Dem steht allerdings auch das Ergebnis gegenüber, dass zwischen $66,4 \%$ und $84,6 \%$ der Bewertungen keine oder lediglich geringe Mängel aufweisen.

\section{Bewertungen auf der Ebene der Einzelmerkmale}

Die Bewertung der Einzelmerkmale durch die Peers erfolgte im Gegensatz zu den vierstufigen Beurteilungen der zusammenfassenden Bewertungen dreistufig: „keine Mängel“/,leichte Mängel“/,gravierende Mängel“. Entsprechend dem Verständnis, dass „leichte Mängel“ noch keinen Hinweis auf drängende Optimierungsbedarfe geben, liegt der Fokus der nachstehenden Darstellungen auf den Einschätzungen der „gravierenden Mängel“.

In Tab. 4 sind jeweils die 5 häufigsten gravierenden Mängel bei den Einzelmerkmalen innerhalb der sechs Bereiche zusammenfassend wiedergegeben.

Insgesamt weisen zwölf von den 67 analysierten Prozessmerkmalen über 15 Prozent gravierende Mängel auf, bei zwei Merkmalen fielen sogar mehr als 25 Prozent der Beurteilungen als „gravierender Mangel“ aus. Besonders stark betroffen sind Einzelmerkmale aus den Bereichen „Diagnostik“ und „Therapieziele und -planung“.

Zusammenfassend zeigt sich, dass die Mängel insbesondere auf zwei Ebenen zu finden sind, die nach dem Selbstverständnis der medizinischen Rehabilitation gerade als „rehaspezifisch" anzusehen sind. Die erste Ebene betrifft die Einbeziehung der subjektiven Perspektive der Patienten in die individuelle Planung und die Bewertung des Reha-Prozesses. Den dargestellten Ergebnissen zufolge ließen sich bei einem essenziellen Anteil der dokumentierten Reha-Prozesse dem subjektiven Krankheitsverständnis, der Selbsteinschätzung zur allgemeinen und zur beruflichen Leistungsfähigkeit und den Plänen des Patienten am Ende der Rehabilitation mehr Gewicht beimessen. Hierin würde sich auch die Umsetzung eines Leitbildes einer gestärkten Patientenrolle wiederfinden.

Die zweite Ebene bezieht sich insbesondere bei Anamnese, Diagnostik und Therapieplanung auf die Berücksichtigung von individuellen Beschwerdekonstellationen der Patienten und einer entsprechenden Abstimmung des erforderlichen therapeutischen Vorgehens. Eine Stärkung dieser Aspekte würde zugleich eine Unterstützung der Rolle der medizinischen Rehabilitation im Versorgungssystem bedeuten, mit individueller Indikationsstellung und Maßnahmenzuweisung.

\section{Zusammenfassung und Diskussion}

Das Qualitäts-Screening innerhalb des Reha-Qualitätssicherungsprogramms der Gesetzlichen Rentenversicherer zielt auf die objektive, reliable und valide Erfassung der Prozessqualität in der medizinischen Rehabilitation. Das hierzu entwickelte Peer-Review-Verfahren basiert auf zufällig ausgewählten Entlassungsberichten, deren Beurteilung anhand einer Checkliste qualitätsrelevanter Prozessmerkmale und eines Manuals durch geschulte, erfahrene und trainierte Rater erfolgt. Das Verfahren zeigte bei den somatischen Rehabilitationsindikationen gute messtechnische Werte und erwies sich als aussagekräftiges und motivierendes Instrument zur vergleichenden Beschreibung und Analyse der in der Klinik ablaufenden Prozesse und des jeweiligen rehabilitativen Versorgungsbereiches. 
In diesem Beitrag ging es um die Anpassung des Peer ReviewVerfahrens an die spezifischen Bedingungen von Patienten mit Abhängigkeitserkrankungen in der stationären Suchtrehabilitation. An einer substanziellen Stichprobe von Entlassungsberichten wurden die Gütekriterien des adaptierten Verfahrens geprüft und gleichzeitig eine Beschreibung des Systems der Suchtrehabilitation durch qualitätsrelevante Prozessmerkmale vorgenommen.

Die Ergebnisse zeigen auf der messtechnischen Ebene, dass das Verfahren den Anforderungen eines Screenings der Prozessqualität für die Suchtrehabilitation gerecht wird:

- Mit einem durchschnittlichen Zeitbedarf von 33 Minuten je beurteiltem Entlassungsbericht erweist sich das PeerReview-Verfahren auch hier als praktikabel. Es ist dennoch weiter über Verkürzungen und zeitsparende Ansätze nachzudenken, auch um die Compliance der als Beurteiler eingesetzten Rehabilitationskliniker dauerhaft sicherzustellen.

- Die Übereinstimmungen zwischen den Ratern sind in den geprüften Bereichen als zufriedenstellend einzuschätzen. Punktuell besteht ein Optimierungsbedarf durch Anpassungen der Schulung sowie durch weitere Präzisierungen der Kategorien und Instruktionen im Manual.

- Die Prüfungen zeigen weiterhin, dass Befürchtungen, einzelne Kliniken würden unter Umständen durch die Zulosung von besonders strengen Gutachtern benachteiligt, nicht zutreffend sind. Die Zufallszuordnung von Berichten zu den Ratern führt zu einem Ausgleich von individuellen Unterschieden der Peers hinsichtlich ihrer Beurteilungsstrenge.

- Die über die Berichte der einzelnen Einrichtungen aggregierten Klinikkennwerte weisen bei den berücksichtigten Dimensionen der Prozessqualität auf deutliche Unterschiede hin. Eine statistisch abgesicherte Differenzierung zwischen Einrichtungen ist aber bei den begrenzten Fallzahlen von Berichten und den damit verbunden hohen Konfidenzintervallen nur eingeschränkt möglich. Bei der Diskussion dieses Sachverhaltes ist allerdings zu bedenken, dass das Peer-Review-Verfahren die Funktion eines Screening-Instrumentes hat, das Hinweise auf mögliche Schwachstellen geben soll. Um diese zu belegen, muss sich, wie bei der Nutzung von Ergebnissen von Screening-Verfahren allgemein üblich, eine systematische und dementsprechend aufwendige Analyse anschließen, diese könnte zum Beispiel durch Klinikbegehung erfolgen.

Insgesamt fand das Verfahren bei den Peers eine hohe Akzeptanz. Dies zeigt auch ein am Ende der Schulung eingesetzter Feedback-Fragebogen: Ein Großteil der Teilnehmer gab an, dass bereits die Schulungen hilfreich für die Qualitätsverbesserung ihrer Entlassungsberichte seien und dass wertvolle Anregungen zur Verbesserung der Prozessqualität in der eigenen Klinik vermittelt wurden. Dabei berücksichtigen die Peers rehabilitationsspezifische Aspekte bewusster, differenzierter und kritischer und fokussieren im Training als Schwachstellen benannte Aspekte genauer. Konkrete Auswirkungen auf die aktuelle Rehabilitationspraxis gehen über Fortbildungen der Mitarbeiter und Anpassungen der Entlassungsberichte hinaus und umfassen auch eine stärkere Berücksichtigung bisher vernachlässigter Aspekte des Rehabilitationsprozesses sowie eine Verbesserung der Dokumentation.
Wer Gelegenheit hatte, die erfahrenen Peers bei den Schulungen in ihren intensiven und teilweise kontroversen Diskussion über die diagnostischen und therapeutischen Erfordernisse einer Suchtbehandlung zu beobachten, wird sich kaum des Eindrucks erwehren, dass das Peer-Review-Verfahren in der Lage ist, den Diskurs um die Qualität zu aktivieren. Wird dieser Prozess von den Peers auch in die Klinik getragen, so ist diese wichtige Zielsetzung des Peer-Review-Verfahrens erreicht.

In der Diskussion des Verfahrens standen als Kritik vor allem drei Argumente im Vordergrund:

- Zeitlicher Aufwand der Peers für die Teilnahme am Verfahren:

Dieser entsteht durch die Vorbereitung zur Versendung der Entlassberichte zur Beurteilung, die Teilnahme an den Schulungen und die Bearbeitung der zu begutachtenden Behandlungsdokumentationen. Durch die zeitliche Einbindung in die Qualitätssicherung zu Lasten der Zeit im beruflichen Alltag ginge ein Teil der ohnehin nur knapp bemessenen Zeit für die therapeutische Arbeit verloren. Dem steht gegenüber, dass die teilnehmenden Kliniken im Gegenzug fundierte Rückmeldungen über wichtige Aspekte in ihrer Prozessqualität erhalten und diese für das interne Qualitätsmanagement nutzbar machen können.

- Steigender Aufwand für die Dokumentation:

Das Wissen um das Qualitäts-Screening erhöht nach dem Eindruck vieler Peers die Ausführlichkeit der Entlassungsberichte.

Innerhalb einer dafür von der Rentenversicherung gebildeten Arbeitsgruppe werden die Erfahrungen aus der Gestaltung des einheitlichen Entlassungsberichtes derzeit geprüft. In diesem Kontext sollen die möglichen Auswirkungen des Peer Reviews auf die Berichtsgestaltung ebenfalls berücksichtigt werden

- Validität des Verfahrens:

Zwar gibt es Hinweise darauf, dass das Peer-Review-Verfahren Qualitätsunterschiede valide abbilden kann. Der eigentliche Prozess der Validierung steht aber noch aus. Hierzu gehören auch Studien, die andere Zugänge der Prozessbeschreibung vergleichend einbeziehen.

Ein spezielles Problem könnte darin liegen, dass die jährlichen Reviews eher die Qualität der Entlassungsberichte als die Behandlungsprozesse verbessern. Da das Verfahren in seiner Zielsetzung für die beteiligten Einrichtungen zunehmend transparenter wird, ist die Hypothese, dass sich mit der Zeit seine Validität partiell verringern könnte, nicht völlig zurückzuweisen. Aber auch dieses Argument belegt lediglich die Notwendigkeit einer steten Weiterentwicklung und Anpassung des Verfahrens.

Andere Fragen im Zusammenhang mit der Validität des Peer Review-Verfahrens ergeben sich auch aus der Handhabung der Ergebnisse durch den jeweiligen Reha-Träger. Unerwünschte Auswirkungen können sich dann ergeben, wenn der jeweils für die Einrichtung zuständige RV-Träger oder der Klinikbetreiber die Ergebnisse ausschließlich im Sinne eines Kontrollinstruments behandelt. In diesem Kontext wäre zu überlegen, welche Vorteile es hätte, den Rückmeldungsprozess über neutrale Instanzen zu organisieren. Ziel wäre es dabei, das Verfahren stärker im Sinne eines Selbst-Management-Instrumentes zu nutzen. 
Im Rahmen der durchgeführten Untersuchung wurde auch eine Beschreibung des Systems der Suchtrehabilitation durch qualitätsrelevante Prozessmerkmale versucht. Sie zeigte, basierend auf 604 Beurteilungen von Entlassungsberichten aus 30 Einrichtungen, dass bei dem Großteil der durchgeführten Behandlungen eine gute Prozessqualität vorzufinden war. Allerdings sind in einigen Bereichen der Suchtrehabilitation auch Schwächen und Optimierungsbedarfe deutlich geworden. Dabei zeigen sich die Bereiche „Diagnostik“, „Therapieziele und -planung“ und die „Sozialmedizinische Stellungnahme“ als besonders betroffen. Hier können sich einerseits direkt aus den Rückmeldungen in die Einrichtungen Verbesserungsansätze ergeben. Auf der anderen Seite bieten auch die Schulungen der Peers eine Möglichkeit, Einfluss auf persönliche Bewertungskriterien zu nehmen und so Veränderungen in der klinischen Praxis anzustoßen.

Abschließend kann man zur Frage der Übertragbarkeit des Peer Review-Verfahrens auf den Indikationsbereich Abhängigkeitserkrankungen zunächst einmal feststellen, dass die Ergebnisse die positiven Ergebnisse aus anderen Indikationsbereichen bestätigen. Allerdings ergeben sich in Bezug auf das Management des Qualitäts-Screenings einige spezifische und noch zu lösende Probleme. So weist ein substanzieller Teil der Kliniken der Suchtrehabilitation nur eine sehr begrenzte Zahl von Behandlungsplätzen auf. Bei den gleichzeitig im Vergleich zu den anderen Rehabilitationsindikationen erheblich längeren Behandlungszeiten in der Suchtrehabilitation ist es nicht ohne weiteres möglich, in dem vorgesehenen Zeitfenster in diesen Kliniken genügend Behandlungsberichte zu erhalten. Hier sind Varianten der Verfahrenssteuerung noch zu entwickeln.

\section{Literatur}

${ }^{1}$ ICIDH International Classification of Impairments, Disabilities and Handicaps. Beta-2 draft, Full Version. Genf: World Health Organisation, 1999

2 Koch U. Entwicklungstrends in der Rehabilitation - Der Beitrag der Rehabilitationswissenschaften und Qualitätssicherung. Deutsche Rentenversicherung 2000; 5: 251-268

${ }^{3}$ Verband Deutscher Rentenversicherungsträger (VDR). Kommission zur Weiterentwicklung der Rehabilitation in der gesetzlichen Rentenversicherung: Abschlussbericht. Frankfurt/Main: VDR, 1991

${ }^{4}$ Müller-Fahrnow W. Zwischenbilanz zur Programmumsetzung Ergebnisse und Perspektiven. Bundesversicherungsanstalt für Angestellte: Rehabilitation. Berlin: BfA 1995: 74-114

${ }^{5}$ Schliehe F. Das Reha-Qualitätssicherungsprogramm der gesetzlichen Rentenversicherung - Perspektiven und Ziele. Deutsche Rentenversicherung 1994; 11: 745-750

${ }^{6}$ Egner U, Gerwinn H, Müller-Fahrnow W, Schliehe F. Das Qualitätssicherungsprogramm der gesetzlichen Rentenversicherung für den Bereich der medizinischen Rehabilitation - Konzept, Stand der Umsetzung und Perspektiven. Die Rehabilitation 1998; (Suppl. 1): S2-S7

${ }^{7}$ Verband Deutscher Rentenversicherungsträger (VDR). Das Qualitätssicherungsprogramm der gesetzlichen Rentenversicherung in der medizinischen Rehabilation - Instrumente und Verfahren. Deutsche Rentenversicherung, DRV-Schriften Band 18. Frankfurt/Main: VDR.

${ }^{8}$ Jäckel WH, Maier-Riehle B, Protz W, Gerdes N. Peer Review: Ein Verfahren zur Analyse der Prozessqualität stationärer Rehabilitationsmaßnahmen. Die Rehabilitation 1997; 4: 224-232
${ }^{9}$ Asendorpf J, Wallbott HG. Maß der Beobachterübereinstimmung: Ein systematischer Vergleich. Zeitschrift für Sozialpsychologie 1979; 10: 243-252

${ }^{10}$ Finn RH. A note on estimating the reliability of categorial data Educational and psychological measurement 1970; 30: 71-76

${ }^{11}$ Maier-Riehle B, Jäckel WH, Gerdes N, Protz W. Übereinstimmung und Unterschiede zwischen Beurteilern bei einem Peer Review-Verfahren. Das Gesundheitswesen 1998; 60: 290-296

\section{Dipl.-Psych. Stephan Kawski}

Forschungsgruppe Qualitätsmanagement Universitätskrankenhaus Hamburg-Eppendorf Abteilung für Medizinische Psychologie Martinistraße 52, Pav. 69 20246 Hamburg

E-mail: kawski@uke.uni-hamburg.de 